\title{
Importancia del desarrollo de un sistema resiliente en el Campus Central del Instituto Tecnológico de Costa Rica
}

Importance of the development of a resilient system in the Central Campus of the Technological Institute of Costa Rica

Sara Guevara-Arce ${ }^{1}$

Guevara-Arce, S. Importancia del desarrollo de un sistema resiliente en el Campus Central del Instituto Tecnológico de Costa Rica. Tecnología en Marcha. Edición especial. Movilidad Estudiantil 6, 2019. Pág 83-92

doi) https://doi.org/10.18845/tm.v32i8.4566 


\title{
Palabras Clave
}

Resiliencia; Planeación Urbana; Rehabilitación Conjunta; Desastres Naturales; Manejo de Daños; Minimización de impacto.

\section{Resumen}

En el siguiente artículo científico se explica la importancia de implementar un plan de desarrollo resiliente en la institución y se busca demostrar las ventajas que puede tener a nivel país. En el proceso de la investigación se realizó una encuesta, con el fin de estudiar la percepción estudiantil ante situaciones desastrosas, dando ellos mismos la respuesta de que estamos más propensos a sufrir desastres naturales por movimientos telúricos y que el lugar más seguro para pernoctar seria el centro de las artes. Luego se pretende suponer el impacto que tendrá establecer una red de protección a nivel localizado, en este caso en el TEC, para a futuro implementarlo a nivel municipal y ojala nacional. El fin de efectuar un plan resiliente nos permite analizar los beneficios a nivel social, estructural, económico y medio ambiental, entre otros. Algunas de las ventajas que supone un plan de evacuación basado en la rehabilitación en conjunto es la pronta restitución de los deberes y del funcionamiento del área que ha sido afectada, ya que la población al tener un conocimiento previo de cómo actuar ante cualquier tragedia ayudará a velar por el orden y a resolver con brevedad el caos a causa de un siniestro. Como se mencionó anteriormente, el trabajo se enfoca en la realización de una encuesta a un porcentaje de estudiantes del campus central del TEC, donde se quiso tomar la percepción de ellos ante la capacidad de resistencia y de organización de la institución, y a la vez valorar la confianza que esta les generar, además de las posibles combinaciones de desastres y el tiempo de respuesta que debería tomarle a la universidad restablecerse posterior a una crisis de este tipo.

\section{Keywords}

Resilience; Urban Planning; Joint Rehabilitation; Natural disasters; Handling of Damage; Minimization of impact.

\begin{abstract}
The following scientific article explains the importance of implementing a resilient development plan in the institution and seeks to demonstrate the advantages that can have at the country level. In the research process, a survey was conducted to study student perceptions of disastrous situations, giving the answer that we are more likely to suffer natural disasters through earthquakes and that the safest place to spend the night would be the Center of the arts. Then it is intended to assume the impact that will have to establish a protection network at a localized level, in this case the TEC, for future implementation at the municipal level. The purpose of making a resilient plan allows us to analyze the social, structural, economic and environmental benefits, among others. Some of the advantages of an evacuation plan based on joint rehabilitation are the prompt restitution of the duties and operation of the area that has been affected, since the population, having prior knowledge of how to act in the face of any tragedy, will help to watch over the order and to solve soon the chaos because of a sinister. As mentioned above, the work focuses on conducting a survey of a percentage of students from the central campus of the TEC, where they wanted to take their perception of the institution's capacity for resilience and organization, while at the same time assessing The confidence that this generate, as well as possible combinations of disasters and the response time that should take the university to re-establish after a crisis of this type.
\end{abstract}




\section{Introducción}

Actualmente las ciudades del mundo están en un constante crecimiento y buscan colocarse con dinamismo en una mejor posición global, para esto necesitan crecer con inteligencia, esto requiere comprometerse con las necesidades de sus habitantes, incrementando la seguridad del entorno en el que viven, usando la tecnología en diferentes ámbitos para asegurar la protección y el eficaz manejo de las situaciones desastrosas a las que podrían estar sometidos en el tiempo.

Hoy por hoy se está introduciendo con intensidad el tema de resiliencia en el área constructiva, más que todo enfocado en el urbanismo, esto busca desarrollar las ciudades de manera analítica y preventiva, para anticipar cómo se actuaría antes, durante y después de una contingencia y así obtener una respuesta eficiente ante esta. El concepto en sí hace referencia a la capacidad de una ciudad expuesta a una amenaza a resistir, absorber, adaptarse y recuperarse de sus efectos de manera oportuna y eficiente [1]. Esto pretende mejorar la respuesta de las ciudades ante eventos catastróficos y así rehabilitar con prontitud el funcionamiento de esta, con tal de no afectar la economía y restablecer el orden cotidiano con brevedad.

El desarrollo resiliente promueve seguridad entre los habitantes, esto es: un sistema que asegure la vida humana, la funcionalidad del sitio después de un evento catastrófico, lo que se traduce en un mejoramiento del sector economía, ya que al invertir en edificaciones resilientes se asegura la buena respuesta de estas ante una catástrofe, dando como resultado el ahorro del recurso monetario dentro del contexto de regeneración de estructuras. Algunas de las ventajas que trae consigo este método según el manual "Como desarrollar ciudades más resilientes" desarrollado por la Oficina de las Naciones Unidas para la Reducción del Riesgo de desastres, UNISDR (por sus siglas en inglés) [2]. son:

- Protección de la vida y bienes muebles, provocando la minimización de muertes.

- Reducción del uso de los recursos urbanos, ante una recuperación o respuesta a los desastres.

- Aumento de confianza de los inversionistas, ya que prevén menos perdidas en caso de catástrofes.

- Ecosistemas equilibrados y centros educativos seguros, lo que aumenta la confianza del estudiante y sus niveles de bienestar en general.

Este último punto genera la idea de adaptar el modelo resiliente en un área reducida y controlada, con tal de observar la respuesta que podría tener la población ante la ejecución del plan y de esta manera ver si es viable aplicarlo a mayor escala, como en ciudades o en el país en el mejor de los casos. Para esto se pretende plantear como iniciar con el desarrollo de un plan resiliente en el área escogida, para luego implementarlo y ponerlo a prueba.

\section{Material y métodos}

La investigación se ejecuta en el campus central del Instituto Tecnológico de Costa Rica (TEC) ubicado en la provincia de Cartago, donde frecuentan su uso estudiantes, profesores, administrativos, personal de mantenimiento, entre otros. Siendo estos la población general, para efectos del estudio se toma como muestra el sector estudiantil del TEC, se aplicó una encuesta digital con el fin de evaluar la percepción de los estudiantes ante eventos catastróficos, donde se recolectó la opinión de aproximadamente el 3,5 por ciento de estos, para un total de 241 respuestas. (Según datos tomados del departamento de admisión y registro que reportó para el segundo semestre del 2016 un número de 7105 estudiantes activos). 
El instrumento de recolección de datos usado fue un formulario de google docs, ya que al estar fuera del país se tuvo la limitación de poder preguntar de manera física. Con este método de evaluación digital se pretende conocer el juicio del estudiante frente a algún siniestro y de esta forma considerar cual es la mayor preocupación, qué lugar le aporta seguridad y así adecuarla de manera resiliente.

Para el análisis de los datos se utilizó la metodología inductiva, que nos permite estudiar hechos individuales, registrarlos y clasificarlos, para así obtener conclusiones universales.

\section{Resultados}

De la encuesta realizada se obtuvieron una cierta cantidad de datos que nos permiten desarrollar un análisis para así plantear soluciones de cómo implementar un plan de resiliencia en la institución, ya que se conoce la opinión de los estudiantes y en base a esto podemos desarrollar el plan alrededor de la comodidad que para ellos generarían los lugares elegidos.

En primera instancia se necesita saber a qué desastre natural creen estar más propensos, para esta encuesta el 55.6\% de la muestra opinó que los movimientos telúricos serian la principal probabilidad de desastre dentro del campus, seguido de un $18.67 \%$ que cree que podría ser el efecto de una erupción del volcán Turrialba, que se encuentra relativamente cerca de la institución, le siguen un $12.45 \%$ que opina que las inundaciones podrían ser la causa y finalmente un $9.96 \%$ para las tormentas y un 3.32\% para huracanes. Los resultados se ilustran en la figura 1.

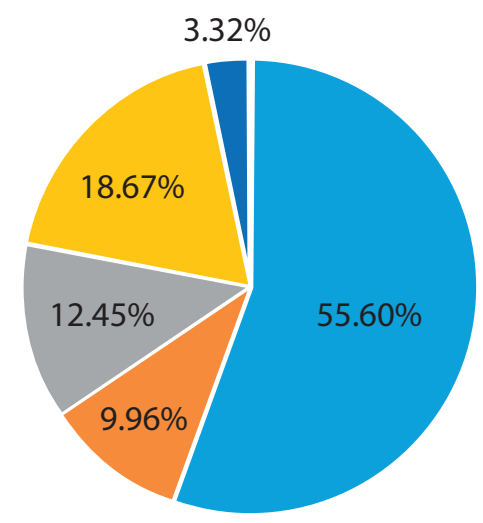

$$
\begin{aligned}
& \text { - Movimientos telúricos } \quad \text { - Tormentas } \\
& \text { - Inundaciones y deslizamientos } \text { - Erupciones } \\
& \text { - Huracán }
\end{aligned}
$$

Figura 1. Qué desastre natural está más propenso a sufrir el Campus Central según los estudiantes.

Después de observar a que creen estar más propensos se necesita saber qué lugar consideran más seguro y que a la vez les genere confianza para poder pernoctar en caso de un evento como los antes mencionados y sus consecuencias. Para los estudiantes la estructura más segura es el auditorio del centro de las artes, le siguen el gimnasio, el comedor y por último la biblioteca, en el caso de otros, los estudiantes señalaron el nuevo edificio de aulas D3 que se encuentra frente a la oficina de ingeniería, la cual obtuvo la misma cantidad de opiniones que la biblioteca (figura 2). 


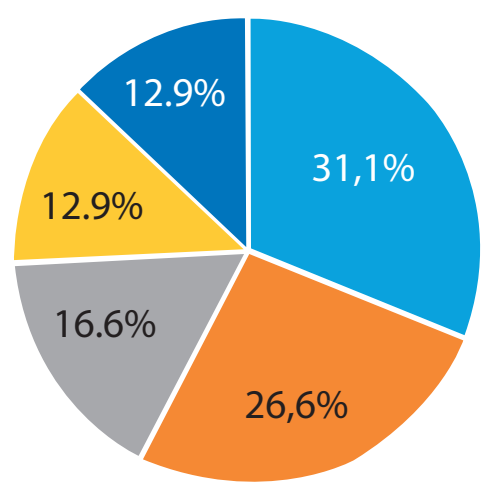

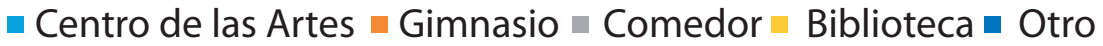

Figura 2. En qué lugar del TEC pernoctaría en caso de un desastre.

Posteriormente se necesita saber que daños consideran aceptables los estudiantes en el caso de un evento catastrófico, ya que con esto podemos evaluar si se sienten seguros dentro de la institución (figura 3). Luego de un siniestro de fuerte magnitud el 66\% de los estudiantes opinan que las instalaciones deberían sufrir daños menores, un 25.3\% aceptarían daños significativos, un $7.9 \%$ que podría estar afectado y un $0.8 \%$ opina que un daño extremo previo al colapso.
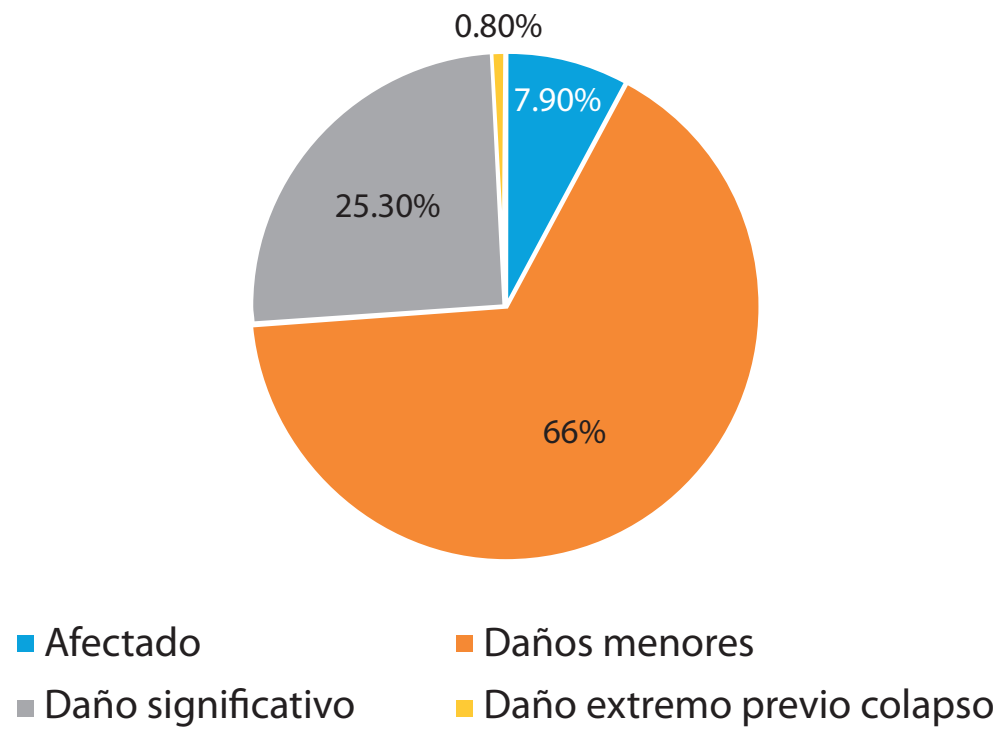

Figura 3. Que daño aceptarían los estudiantes.

Luego se evaluó que posible evento podría darse luego de un movimiento telúrico, como consecuencia de este o afectación de otro desastre en el peor de los casos. Para esto obtuvimos que la peor combinación según los estudiantes en orden de importancia seria: Primero que se desate una erupción con un $26.97 \%$, le sigue un $26.14 \%$ que opina que un incendio, un $24.07 \%$ teme a las fugas de gas, y en menor proporción se obtuvieron porcentaje de un $6.64 \%$ en explosión, 3.73\% colapso de edificios, $2.49 \%$ deslizamientos, $6.22 \%$ todos los mencionados y un $2.49 \%$ otros opino que otros, que se explicarán en el análisis de datos como corresponde (figura 4). 


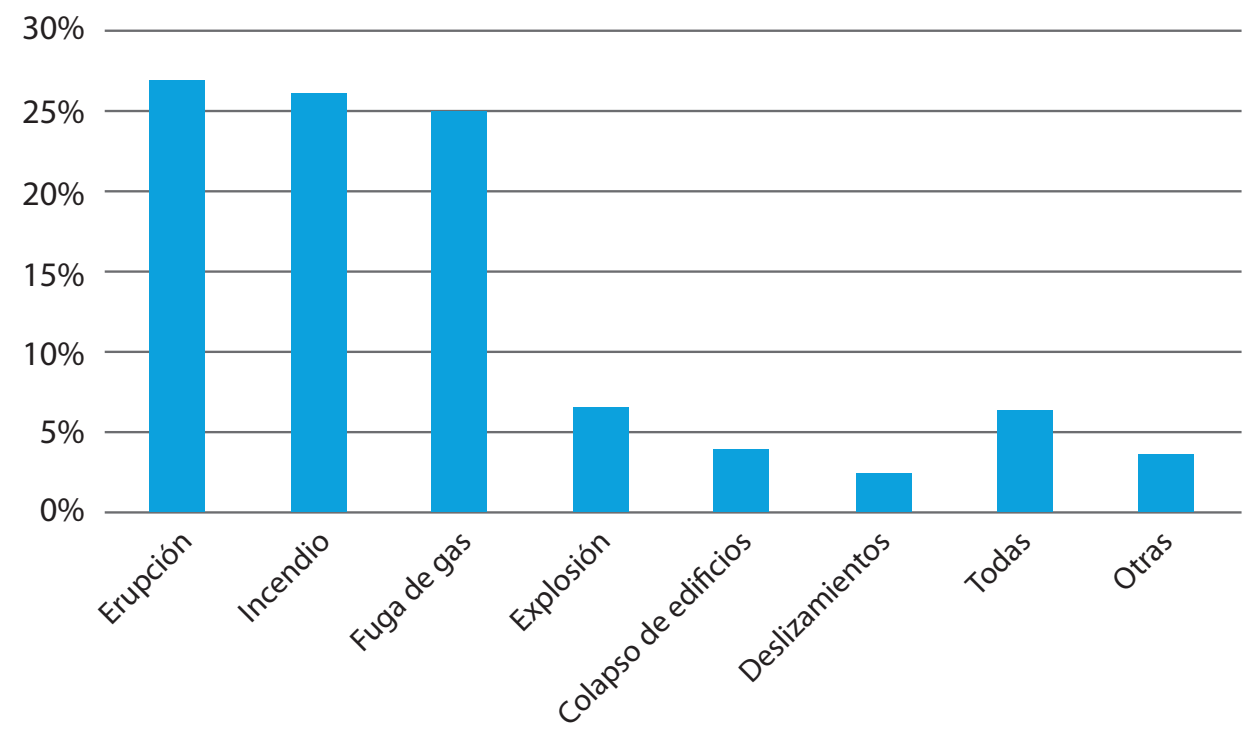

Figura 4. Eventualidades que consideran los estudiantes que podrían ocurrir luego de una catástrofe.

También es importante indagar en cuanto tiempo esperan los estudiantes que los encargados de la institución restablezcan labores después los daños causados por el o los eventos que puedan ocurrir, tomando en cuenta que estos sean catastróficos. Como se indica en la figura 5 el $30.3 \%$ de la muestra opina que un día debe ser suficiente, mientras que el $24.5 \%$ piensa que dos días, los demás porcentajes van asociados con tiempos más largos o cortos, por ejemplo $19.1 \%$ cree que debería tomar una semana, un $6.6 \%$ opina que tres horas, un $3.7 \%$ se inclina por medio día y un $15.8 \%$ indica que plantearían tiempos distintos a los propuestos.

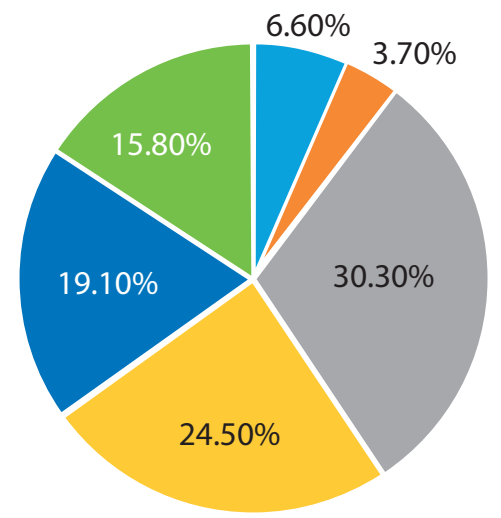

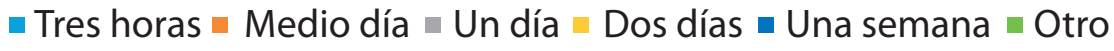

Figura 5. Tiempo que los estudiantes consideran necesario para restablecer labores.

Subsiguientemente se valoraron los servicios básicos que los estudiantes consideran que deberían resistir o restablecerse con mayor brevedad, para esto se colocaron cinco servicios que se debían calificar del uno al cinco en orden de importancia tomando el uno como menos importante y el cinco como primordial, para así puntuar el orden de trascendencia para ellos. De los resultados generados por la muestra se obtuvo que el servicio principal debe ser el agua con un $84.2 \%$ de coincidencia, le siguen en orden, la telefonía $45.6 \%$, la electricidad $42.7 \%$, los drenajes $32 \%$ y de ultimo el internet $14.9 \%$. 
Otro punto importante es saber si el estudiante sabe qué hacer antes, durante y después de que ocurra alguna catástrofe, como se muestra en la figura 6 el 60.2\% cree saber cómo manejar la situación mientras un $32 \%$ está seguro y el restante $27 \%$ no tiene idea de cómo responder ante una crisis de este tipo.

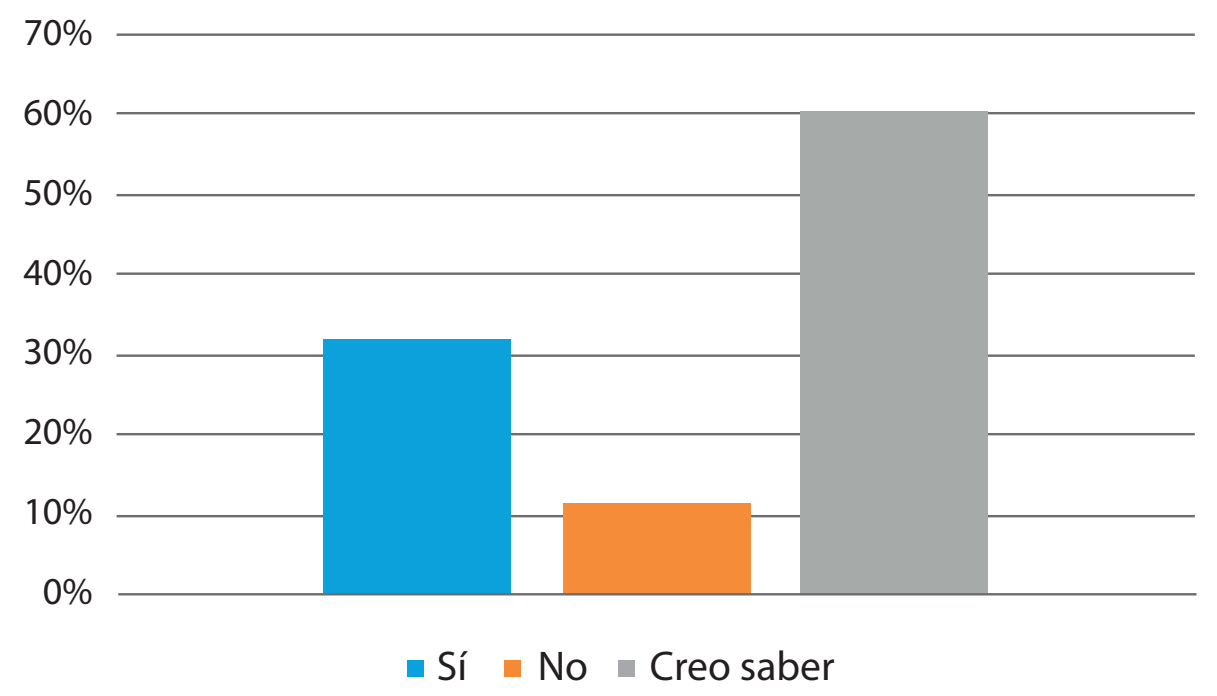

Figura 6. Saben que hacer antes, durante y después de un evento catastrófico.

Para finalizar la encuesta se tomó el criterio de los estudiantes sobre como consideran la capacidad de organización de la institución ante desastres naturales y que tan confiable es para ellos el manejo de estos. El $41.5 \%$ cree que es buena, el $36.5 \%$ regular un $9.1 \%$ cree que es deficiente, el $7.5 \%$ no responden ya que no tienen ningún parámetro para guiarse, el $4.1 \%$ cree que es excelente y un $1.2 \%$ opina que es mala (figura 7 ).

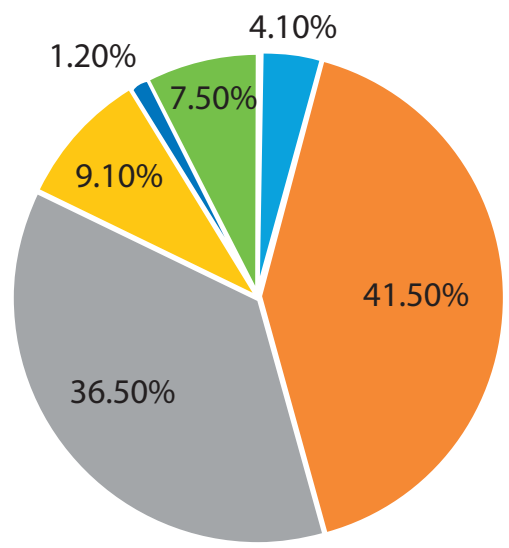

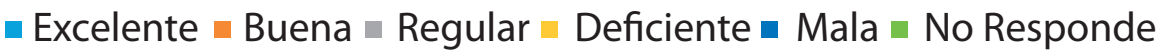

Figura 7. Como consideran la capacidad de organización del TEC ante una contingencia. 


\section{Discusión}

El análisis de estos datos nos permite desarrollar una solución para la problemática elegida: la ausencia de un plan resiliente en la institución, para esto se explicarán primeramente los resultados obtenidos de la encuesta. En la primera pregunta realizada, vemos que los estudiantes opinan en su mayoría que los movimientos telúricos podrían ser la mayor causa de algún evento catastrófico, esto debido a que Costa Rica es un país sumamente sísmico, también vemos que un pequeño porcentaje indica que los huracanes podrían afectar el campus central, el criterio de esta fracción de estudiantes puede estar vinculada a la reciente presencia del Huracán Otto en nuestro país, ya que el territorio no sufre con frecuencia eventos de este tipo.

La segunda pregunta consulta que zona de la institución consideran los alumnos más seguro para tomar como albergue, esto nos proporciona el lugar en donde deberíamos desarrollar o implementar el plan de evacuación, ya que según la muestra denota seguridad y confianza. La población estudiantil podría ser alojada ahí mientras se desarrollan las maniobras de evacuación necesarias o el restablecimiento de los servicios básicos de la institución.

Para la tercera pregunta los criterios de evaluación de daños se tomaron de las normas estandarizadas FEMA [3] de los Estados Unidos de América, esto con tal de obtener un parámetro certero y establecido del análisis de daños. Los estudiantes en su mayoría expresan que estarían de acuerdo con que la Universidad sufriera daños menores ante la consideración de que se está evaluando un evento catastrófico, esta pregunta refleja la confianza del estudiantado en la estructura que compone al TEC, pero a la vez genera la necesidad de una rápida respuesta y solución ante algún siniestro de parte de las autoridades de la institución.

La cuarta pregunta tiene como fin examinar que posibles combinaciones de eventos se podrían dar luego de un desastre natural o en este caso un movimiento telúrico, se escoge este como primera opción ya que Costa Rica es un país sísmicamente activo, al estar ubicado sobre una zona de subducción de placas tectónicas. El fin de indagar las posibles combinaciones de eventos nace de examinar el caso del terremoto de Japón del 2011 en donde como consecuencia de este se genera un tsunami y a los pocos días se ven afectados por un frente frío y fuertes nevadas. En el caso particular del TEC al estar relativamente cerca del volcán Turrialba, una posible mezcla de eventos sería la expulsión de ceniza, aunque propiamente en el Campus, la mayor probabilidad podría ser generada por incendios o explosiones, ya que muchas de las zonas manejan gas, como lo son el comedor y los laboratorios dispersos en las diferentes facultades y Escuelas. La población también plantea los deslizamientos, que podrían provocarse por fuertes lluvias, pero este caso puede ser bastante improbable por la localización de la Universidad. Los estudiantes también opinaron en menor cantidad que podrían sufrir otros daños, en este caso algunos de los denotados fueron fugas de agua, réplicas y derrames de químicos.

La quinta pregunta demuestra lo comentado en el punto tres, el tiempo de respuesta esperado por los estudiantes es bastante estricto para tratarse de una catástrofe, ya que la mayoría opina que uno o dos días deberían ser suficientes para restablecer las labores y el normal funcionamiento de la institución. Lo que puede ser complicado dependiendo de la magnitud del evento al que esté sometida la zona.

El estudiantado espera que luego de una catástrofe la mayoría de servicios básicos resistan, o que su funcionamiento se active a mayor brevedad posible, al encuestar el orden de importancia en los servicios básicos obtenemos que el agua y la telefonía son primordiales para estos, el primero por la salubridad y el segundo porque muchos son originarios de zonas rurales o viven temporalmente en Cartago debido a su periodo de estudio en la institución, por lo tanto, poder incorporar la comunicación luego de eventos de esta índole genera bienestar para los perjudicados y sus familias, ya que pueden dar información de su estado y del escenario 
que se vive. Es trascendental mantener actualizada de la situación a la población afectada, el desconocimiento genera intranquilidad y con esto caos lo que ralentiza el proceso y puede entorpecer las labores de reposición.

Al finalizar la evaluación se debe tomar en cuenta cómo considera el estudiante la capacidad propia y de la institución respecto a la preparación ante algún evento catastrófico, por ejemplo si el joven sabe o no como actuar en el momento y posterior a este, la mayoría de ellos cree que saben cómo hacerlo, pero ciertamente los simulacros en la institución son escasos por lo que no se puede asegurar esto. Se debe considerar como opción el proponer capacitaciones al estudiante para mejorar su técnica y tiempo de respuesta ante alguna tragedia. En cuanto a la organización de la institución la muestra encuestada califica esta entre buena y regular, lo que supone que no hay plena confianza en la capacidad de respuesta de los encargados de evacuación del TEC.

El desarrollo de un plan resiliente lo que propone es el restablecimiento en conjunto de la parte estructural y social, esto con tal de lograr la pacificación de los afectados y la continuidad de las labores. A la hora de crear los planes, se debe tomar en cuenta algunos aspectos como lo son:

- Realizar un análisis de riesgos y combinación de estos.

- Analizar en pequeña y gran escala a que está expuesto el campus luego de un desastre natural.

- Incluir estudiantes, personal administrativo y de mantenimiento en los programas de evaluación de riesgos y evacuación de la institución.

- Plantear el plan de acción.

- Asignar a los encargados de mantenimiento el estudio correspondiente para lograr que los servicios básicos resistan o como recuperarlos con prontitud luego de una catástrofe.

- Establecer comisiones de trabajo para trasladar a las personas al sitio establecido de pernoctación.

- Incrementar los simulacros, al menos dos por semestre.

- Formar grupos preferiblemente de profesores con conocimientos de estructuras y seguridad para darle seguimiento y supervisión al plan establecido.

Además dentro de estos se deben formar equipos de trabajo para evaluar el daño sufrido por las estructuras luego del evento. De esta manera se podría mejorar la percepción de los estudiantes ante la capacidad organizativa del centro universitario y la respuesta de la institución ante alguna catástrofe.

\section{Conclusión}

1. Tomando en cuenta que la percepción de los estudiantes ante la capacidad de la universidad de manejar de forma adecuada un siniestro fue baja, la implementación de una red de protección o rehabilitación en conjunto en la institución y tomar en consideración establecer en el centro de las artes como el principal albergue del campus y acondicionarlo para cubrir las necesidades podría mejorar la confianza del estudiante. 
2. Con el desarrollo de un plan resiliente a pequeña escala, se busca interesar a los gobiernos locales para que efectúen estos en sus municipios y convertirlos en objetivos primordiales de los planes políticos, esto con tal de perseguir el bienestar público.

3. La importancia de realizar un plan a micro escala como lo es el presente trabajo de investigación es buscar crear conciencia a nivel nacional, ya que esto podría impactar de manera positiva en muchos aspectos, pero se necesita el compromiso del gobierno para invertir la cantidad necesaria de dinero y así hacer edificios con mayor capacidad de resistencia, con esto se obtendría en el ámbito:

- Social: un riesgo menor en la pérdida de vidas.

- Estructural: conservación de la comunicación con las ciudades cercanas o edificaciones de importancia como lo son los hospitales y albergues.

- Económico: minimización del impacto monetario luego de una catástrofe, al lograr reducir los gastos de reparación y regeneración de edificaciones.

- Medio ambiental: reducción de la contaminación al minimizar la reparación de estructuras, disminución de carbono en la atmosfera, sonido en el ambiente, polvo y residuos de materiales en el aire, entre otros.

4. La construcción de edificios resilientes proporciona seguridad tanto a la ciudadanía como a los inversionistas, que suministrarían mayor capital para la generación de estructuras, logrando así la planificación urbana adecuada para rehabilitarse con prontitud.

\section{Referencias}

[1] X, Jiménez. Resiliencia urbana, una nueva mirada sobre las ciudades [Online]. Más digital, 2012. Disponible en: http://blogs.elperiodico.com/masdigital/afondo/resiliencia-urbana-una-nueva-mirada-sobre-las-ciudades

[2] Cómo desarrollar ciudades más resilientes, 1ra ed. Ginebra: Helena Molin Valdés, UNISDR, 2012, pp. 15, 16.

[3] Damage Assessment Operations Manual. Federal Emergency Management Agency, Washington, D.C. 2016, pp. $69,70$. 